\title{
Paediatric Crossword Puzzle -1 (Solution)
}

Manouri P. Senanayake ${ }^{1}$

Sri Lanka Journal of Child Health, 2008; 37: 102

\begin{tabular}{|l|l|l|l|l|l|l|l|l|l|l|l|l|}
\hline $\mathbf{P}$ & $\mathbf{R}$ & $\mathbf{O}$ & $\mathbf{S}$ & $\mathbf{T}$ & $\mathbf{A}$ & $\mathbf{G}$ & $\mathbf{L}$ & $\mathbf{A}$ & $\mathbf{N}$ & $\mathbf{D}$ & $\mathbf{I}$ & $\mathbf{N}$ \\
\hline $\mathbf{W}$ & & & $\mathbf{A}$ & $\mathbf{N}$ & $\mathbf{A}$ & $\mathbf{E}$ & $\mathbf{M}$ & $\mathbf{I}$ & $\mathbf{A}$ & & & $\mathbf{O}$ \\
\hline $\mathbf{P}$ & & $\mathbf{V}$ & $\mathbf{P}$ & & & & & $\mathbf{D}$ & & $\mathbf{S}$ & & $\mathbf{N}$ \\
\hline $\mathbf{W}$ & $\mathbf{A}$ & $\mathbf{S}$ & $\mathbf{H}$ & $\mathbf{O}$ & $\mathbf{U}$ & $\mathbf{T}$ & & $\mathbf{O}$ & $\mathbf{X}$ & $\mathbf{Y}$ & & $\mathbf{A}$ \\
\hline & $\mathbf{N}$ & & & $\mathbf{C}$ & & $\mathbf{A}$ & & $\mathbf{S}$ & & $\mathbf{D}$ & $\mathbf{O}$ & $\mathbf{T}$ \\
\hline & $\mathbf{G}$ & $\mathbf{E}$ & $\mathbf{N}$ & $\mathbf{U}$ & & $\mathbf{K}$ & & $\mathbf{I}$ & $\mathbf{G}$ & $\mathbf{E}$ & & $\mathbf{E}$ \\
\hline $\mathbf{P}$ & $\mathbf{L}$ & $\mathbf{A}$ & $\mathbf{N}$ & $\mathbf{O}$ & & $\mathbf{Y}$ & & & $\mathbf{D}$ & $\mathbf{H}$ & $\mathbf{A}$ & \\
\hline & $\mathbf{M}$ & $\mathbf{A}$ & $\mathbf{G}$ & & $\mathbf{T}$ & $\mathbf{A}$ & $\mathbf{P}$ & $\mathbf{E}$ & & $\mathbf{A}$ & & $\mathbf{F}$ \\
\hline & $\mathbf{A}$ & & & $\mathbf{E}$ & & $\mathbf{S}$ & & & & $\mathbf{M}$ & $\mathbf{C}$ & $\mathbf{H}$ \\
\hline
\end{tabular}

${ }^{1}$ Professor in Paediatrics, Faculty of Medicine, University of Colombo 